\title{
Increased Rac1 activity and Pak1 overexpression are associated with lymphovascular invasion and lymph node metastasis of upper urinary tract cancer
}

\author{
Takao Kamai¹ , Hiromichi Shirataki², Kimihiro Nakanishi' , Nobutaka Furuya'1, Tsunehito Kambara' ', Hideyuki Abe1, \\ Tetsunari Oyama ${ }^{3}$ and Ken-Ichiro Yoshida ${ }^{1}$
}

\begin{abstract}
Background: Lymphovascular invasion (LVI) and lymph node metastasis are conventional pathological factors associated with an unfavorable prognosis of urothelial carcinoma of the upper urinary tract (UC-UUT), but little is known about the molecular mechanisms underlying LVI and nodal metastasis in this disease. Rac1 small GTPase (Rac1) is essential for tumor metastasis. Activated GTP-bound Rac1 (Rac1 activity) plays a key role in activating downstream effectors known as Pak (21-activated kinase), which are key regulators of cytoskeletal remolding, cell motility, and cell proliferation, and thus have a role in both carcinogenesis and tumor invasion.

Methods: We analyzed Rac1 activity and Pak1 protein expression in matched sets of tumor tissue, non-tumor tissue, and metastatic lymph node tissue obtained from the surgical specimens of 108 Japanese patients with UC-UUT.

Results: Rac1 activity and Pak1 protein levels were higher in tumor tissue and metastatic lymph node tissue than in non-tumor tissue (both $P<0.0001$ ). A high level of Rac1 activity and Pak1 protein expression in the primary tumor was related to poor differentiation $(P<0.05)$, muscle invasion $(P<0.01)$, LVI $(P<0.0001)$, and lymph node metastasis $(P<$ 0.0001). Kaplan-Meier survival analysis showed that an increase of Rac1 activity and Pak1 protein was associated with a shorter disease-free survival time $(P<0.01)$ and shorter overall survival $(P<0.001)$. Cox proportional hazards analysis revealed that high Rac1 activity, Pak1 protein expression and LVI were independent prognostic factors for shorter overall and disease-free survival times $(P<0.01)$ on univariate analysis, although only Pak1 and LVI had an influence $(P<$ $0.05)$ according to multivariate analysis.
\end{abstract}

Conclusions: These findings suggest that Rac1 activity and Pak1 are involved in LVI and lymph node metastasis of UCUUT, and may be prognostic markers for this disease.

\section{Background}

Urothelial carcinoma of the upper urinary tract (UCUUT) is relatively uncommon, accounting for $<10 \%$ of all urothelial malignancies, but its incidence is increasing [1]. Many patients who undergo curative resection develop systemic metastases within a few years, so the prognosis of this cancer is poor [2], presumably due to occult micrometastasis at the time of surgery because of the thin walls and rich lymphatic drainage of the ureter.

\footnotetext{
* Correspondence: kamait@dokkyomed.ac.jp

1 Department of Urology, Dokkyo Medical University, Mibu, Tochigi, Japan Full list of author information is available at the end of the article
}

Metastasis involves the spread of tumor cells from the primary tumor to a distant site [3], and is the major cause of human cancer death. Various pathological studies have shown that poorly differentiated cancer, muscle invasion, lymph node metastasis, and lymphovascular invasion (LVI) are associated with recurrence and are unfavorable prognostic factors for UC-UUT $[2,4,5]$. Thus, LVI and lymph node metastasis are used to predict the prognosis. Despite their clinical importance, little is known about the molecular mechanisms of LVI and lymph node metastasis, making it important to examine the factors 
playing a role in LVI and lymph node metastasis of UCUUT.

Members of the Rho small GTPases family, prototype RhoA, Rac1, and Cdc42, are involved in the regulation of a variety of cellular processes, such as organization of the microfilament network, cell-cell contact, and malignant transformation, and also perform essential and specialized functions during organization of the actin cytoskeleton [6]. RhoA regulates the formation of stress fibers and focal adhesions in cells, while Rac1 regulates the formation of lamellipodia and membrane ruffling, and Cdc42 regulates the formation of filopodia [6,7]. In addition, a number of investigations have established a significant role of GTPases from the Rho family in several human tumors, including UC-UUT $[7,8]$. Rac1 is ubiquitously expressed and exists in two conformational states. In response to extracellular signals, interconversion of these two states occurs via guanine nucleotide exchange factors (GEFs), which convert the inactive GDP-bound form of Rac1 to its active GTP-bound form, while GTPase-activating proteins (GAPs) inactivate proteins (GAPs) inactivate Rac1. After activation, Rac1 interacts with various specific effectors to coordinate the activation of a multitude of signaling cascades that influence diverse physiological outcomes. The Pak (p21-activated kinase) serine/ threonine kinases have recently been found to be key regulators of cytoskeletal remolding, cell motility, and cell proliferation, with a role in both carcinogenesis and cellular invasion [9]. It has been reported that Pak1, the best characterized member of this family, shows increased expression and activity in human cancers [9-11]. Multiple signalling pathways converge to promote activation of Pak1 through both small GTPases and several of the tyrosine kinases. In turn, activated Pak1 regulates diverse cellular functions. Pak1 binds to Rac1 in a GTP-dependent manner, after which activated Pak1 regulates cellular functions such as cytoskeletal dynamics, cell adhesion, and transcription [9]. Rac1 signals through Pak1 to activate c-Jun N-terminal kinase (JNK) [9], placing Rac1 between the Ras small GTPases (Ras) and mitogen-activated protein kinase (MAPK) in the signaling cascade from growth factor receptors and v-Src to activation of JNK $[12,13]$. Gao et al. reported that a low molecular weight inhibitor of Rac GTPase targeting the activation of Rac by GEF was able to reverse the tumor cell phenotype associated with deregulation of Rac [14]. In addition, several low molecular weight inhibitors have been shown to interfere with Pak kinase activity or function [9]. These findings suggest that Rac1 and Pak may be potential molecular targets for the treatment of cancer.

Regarding the expression of Rho family GTPases in human cancers, most previous reports were based on the investigation of protein expression levels by Western blotting and immunohistochemistry. However, only the active GTP-binding form (active GTPase) recognizes target proteins and generates a response. Increased activity of Rac1 and overexpression of Pak1 are associated with the progression of cancer, but most of the evidence has come from cell culture studies. Therefore, the role of active Rac1 GTPase and its downstream effector needs to be studied by using surgically resected samples of human tumors to better assess their contribution to human cancer. Accordingly, we examined the expression of active GTP-bound Rac1 (Rac1 activity) and its downstream effector Pak1 in the primary tumors and metastatic lymph nodes of patients with UC-UUT by Western blotting, and also assessed the relation of these molecules with clinicopathological features. There have been few reports about the simultaneous analysis of Rac1 and Pak1 in human tumor tissues. Such information could be useful for developing individualized treatment strategies and could potentially improve the design and application of adjuvant therapy for UC-UUT.

\section{Methods \\ Patients and tissues}

Between 1995 and 2006, surgical specimens of UC-UUT were obtained from 108 consecutive Japanese patients (77 men and 31 women) aged 42 to 89 years (mean age: 71.9 years) with newly diagnosed primary transitional cell carcinoma (TCC) of the renal pelvis and ureter without distant metastasis $\left(\mathrm{cT}_{\text {any }} \mathrm{N}_{\text {any }} \mathrm{M} 0\right)$. These specimens were reviewed in the present study. All patients routinely underwent imaging investigations (CT and/or MRI) before surgery to acquire information for staging. The follow-up time ranged from 5 to 132 months, with a median follow-up period of 41 months. Patients underwent surgery before receiving any other therapy.

During nephroureterectomy, the patients also underwent lymphadenectomy when enlarged lymph nodes were confirmed. In all patients, tumor tissue, non-tumor tissue, and lymph node tissue were acquired from the resected specimens after removing excess stromal tissue. Then the tissues were embedded in OCT tissue compound (Miles, Elkhart, IN, USA) and stored at $-80^{\circ} \mathrm{C}$ according to the method described previously [15]. The grade and stage of each tumor were classified according to the TNM system [16]. This study was conducted in accordance with the Helsinki Declaration and institutional review board approval in Dokkyo Medical University Hospital was obtained. In addition, each patient signed a consent form approved by the Committee on Human Rights in Research of our institution.

Systemic chemotherapy with methotrexate, vinblastine, Adriamycin, and cisplatin (M-VAC) was given to the 16 patients who had lymph node metastasis at nephroureterectomy, as well as those in whom lymph node metasta- 
sis (14 patients) or distant metastasis (5 patients) was detected postoperatively [17].

\section{Rac1 activation assay and Western blotting}

Tumor tissue and normal tissue specimens were carefully dissected free of stromal tissue. To measure Rac1 activation, we performed a Rho-binding domain (RBD) affinity precipitation assay for Rac1-GTP using a specific Rac1 antibody according to the manufacturer's protocol (Cytoskeleton, BK126, Denver, CO) [18-20]. Tissue extracts were obtained from $50 \mathrm{mg}$ of each type of tissue and the protein concentration was determined by Bradford's method (BIO-RAD, Hercules, CA). Then the extracts were equalized with ice cold cell lysis buffer containing $25 \mathrm{mM}$ Tris (pH 7.5), $10 \mathrm{mM} \mathrm{MgCl}_{2}, 0.5 \mathrm{M} \mathrm{NaCl}$, and $1 \%$ Triton $\mathrm{X}-100$ to obtain identical protein concentrations. Next, equivalent amounts of protein were added to $15 \mu$ l of Pak (p21-activated kinase)-RBD beads and incubated at $4^{\circ} \mathrm{C}$ on a rotor for $1 \mathrm{hr}$. Then the Pak-RBD beads solution was centrifuged at 3,000 $\mathrm{g}$ for $1 \mathrm{~min}$ at $4^{\circ} \mathrm{C}$ and supernatant was removed. After washing the pellet three times, the bound proteins were analyzed by Western blotting, as described previously [21]. Briefly, $25 \mu \mathrm{g}$ of GTP-Rac1 bound to Pak-RBD beads was separated by SDS-PAGE (5-20\% gradient gel) and electrotransferred to a polyvinylidene difluoride membrane (Sequi-Blot PVDF membranes; BIO-RAD). Rac1 His-tagged protein (100, 50 , and $10 \mathrm{ng} / \mu \mathrm{l}$ ) was also run on the gel as a standard. If the intensity of the immunoreactive band was outside the range of $10-100 \mathrm{ng} / \mu \mathrm{l}$, samples were diluted with loading buffer and run on the gel again. Total cell lysate with GDP was employed as a negative control. After membranes were blocked to eliminate nonspecific binding, membrane-bound proteins were probed with an anti-Rac1 monoclonal antibody (Cytoskeleton, BK126). Then the membranes were washed and incubated with a horseradish peroxidase-conjugated secondary antibody. Antibody-bound protein bands were visualized by chemiluminescence, the blotted membrane was subjected to densitometry by scanning with a ChemiDoc XRS-J imaging scanner (BIO-RAD), and the data were analyzed with NIH Image software. The mean value for three experiments was obtained with each tissue specimen.

For measurement of Pak1, $50 \mu \mathrm{g}$ of cytosolic protein was separated by SDS-PAGE $(12.5 \%$ gel $)$ and electrotransferred to a polyvinylidene difluoride membrane (Immobilon-P membrane; Millipore, Bedford, MA). After the membrane was blocked, the bound proteins were probed with specific antibodies (Santa Cruz Biotechnology; sc-881, Santa Cruz, CA) and a primary antibody for beta actin (Santa Cruz Biotechnology, Santa Cruz, CA). Hela cells were used as a positive control for Rac1 and Pak1 expression. Next, the membranes were washed and then incubated with horseradish peroxidase-conjugated secondary antibodies. Antibody-bound protein bands were visualized by chemiluminescence, the blotted membrane was scanned for densitometry with a PDI imaging scanner (Agfa Japan, Tokyo), and the data were analyzed with NIH Image software. Expression of active Rac1 and Pak1 was determined relative to that of beta actin in the tumor tissue and corresponding normal tissue specimens, after which relative expression was calculated. For quantification of these proteins, the relative amount of Rac1 or Pak1 in tumor tissue was expressed as a ratio of the optical density of the band obtained from the tumor specimen to that from the corresponding normal tissue (which was set at 1.0 ) by densitometric analysis as described previously [21-23]. The mean values for tumor tissue and non-tumor tissue were calculated from three experiments [21,22].

\section{Immunohistochemistry}

To support the data obtained by Western blotting, immunohistochemistry was performed with the same antibodies utilized for Western blotting on 3 tumors from patients with lymph node metastases $(\mathrm{pN}+)$ and 5 tumors from patients without nodal metastasis $(\mathrm{pN}-)$, as described previously [20-22].

\section{Statistical analysis}

Western blotting data were analyzed by the Mann-Whitney $U$ test to compare two groups [21,22], or by the Kruskal-Wallis test for comparisons among three or more groups. Spearman's rank correlation coefficient analysis was employed to determine the relation between Rac1 activity and Pak1 expression. Rac1 activity and Pak1 expression, as well as tumor grade, $\mathrm{pT}$ stage, lymph node metastasis, and LVI, were assessed for their relation to disease-free survival and overall survival by univariate and multivariate analyses with the Cox proportional hazards model. The Kaplan-Meier method was used to estimate survival, and differences of survival were assessed by the log-rank test. Probability values of less than 0.05 were considered significant. Data were analyzed with commercially available software.

\section{Results}

\section{Association of Rac1 activity and Pak1 expression with tumor characteristics}

Rac1 and Pak1 proteins were detected in tumor tissues, non-tumor tissues, metastatic lymph nodes, and normal lymph nodes (Figures. 1, 2). The level of active (GTPbound) Rac1 was significantly increased in tumor tissues $($ mean \pm S.D. $=2.72 \pm 1.72)$ and metastatic lymph nodes $(1.86 \pm 0.34)$ compared with the level in non-cancerous tissues, which was set at 1.0 [21-23], $(\mathrm{P}<0.0001$, Figure. $3 \mathrm{~A})$. An increase of Rac1 activity in primary tumors was 


\section{Rac1}

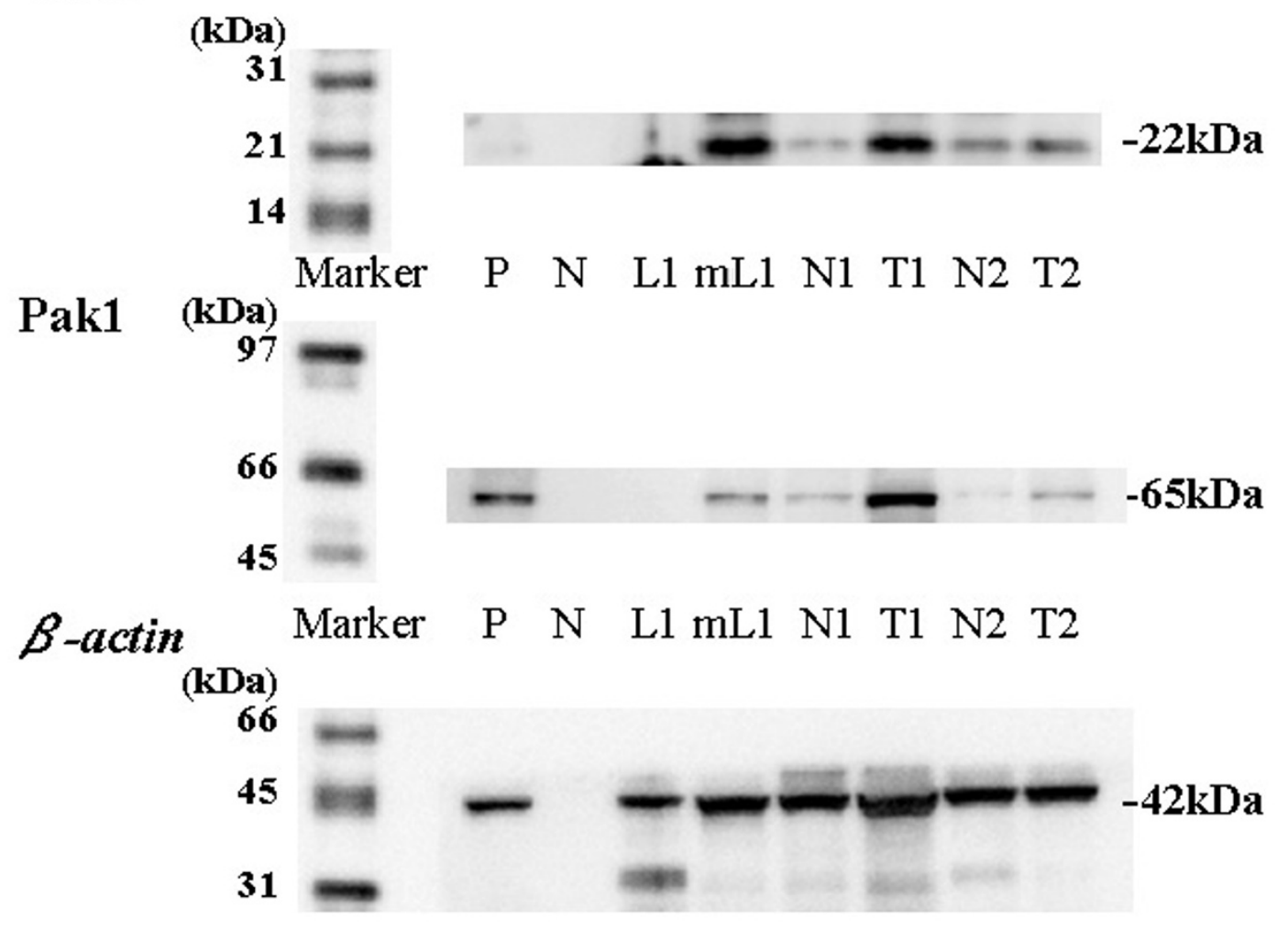

$\begin{array}{llllllllll}\text { Marker } & \mathrm{P} & \mathrm{N} & \mathrm{L} 1 & \mathrm{~mL} 1 & \mathrm{~N} 1 & \mathrm{~T} 1 & \mathrm{~N} 2 & \mathrm{~T} 2\end{array}$

Figure 1 Expression of GTP-binding (active form) Rac1 (22 kDa), Pak1 (65 kDa) and beta actin (42 kDa) proteins using Western blotting. M; marker, P; positive control using Hela cells, N; negative control, T1,2; tumor tissue, N1,2; non-tumor tissue. LN1; non-tumor lymph node, mLN1; metastatic lymph node, Each number corresponds to a case number.

associated with poorly differentiated cancer (grade $1 ; 2.38$ \pm 1.38 , grade $2 ; 2.33 \pm 1.31$, grade $3 ; 3.30 \pm 2.08, \mathrm{P}=$ 0.0471 , Figure. $3 \mathrm{~B})$, local invasion ( $<\mathrm{pT} 1 ; 2.38 \pm 1.35$, pT2; $2.32 \pm 1.31, \mathrm{pT} 3 ; 2.91 \pm 1.69$, pT4; $4.46 \pm 3.14, \mathrm{P}=0.1417$, Figure. 3 C), lymph node metastasis (pN0; $2.55 \pm 1.62$, pN1-3; $3.52 \pm 1.96, \mathrm{P}<0.05$, Figure. 3D), and LVI (LVI(-); $2.04 \pm 0.97, \mathrm{LVI}(+) ; 3.40 \pm 2.02, \mathrm{P}<0.0001$, Figure. $3 \mathrm{E})$.

The level of Pak1 protein was significantly higher in tumor tissues (mean \pm S.D. $=2.68 \pm 1.26$ ) and metastatic lymph nodes $(2.77 \pm 1.60)$ than the level in non-cancerous tissues, which was also set at 1.0 [21-23], $(\mathrm{P}<0.0001$, Figure. 4A). Higher expression of Pak1 protein in the primary tumor was associated with poorly differentiated cancer (grade $1 ; 1.67 \pm 0.34$, grade $2 ; 2.38 \pm 0.96$, grade 3 ; $3.29 \pm 1.45, \mathrm{P}<0.0001$, Figure. $4 \mathrm{~B})$, local invasion $(<\mathrm{pT} 1$; $1.89 \pm 0.60, \mathrm{pT} 2 ; 2.52 \pm 0.97, \mathrm{pT} 3 ; 3.09 \pm 1.12, \mathrm{pT} 4 ; 4.56 \pm$
2.10, $\mathrm{P}<0.0001$, Figure. $4 \mathrm{C}$ ), lymph node metastasis (pN0; $2.43 \pm 1.09$, pN1-3; $3.84 \pm 1.35, \mathrm{P}<0.0001$, Figure. $4 \mathrm{D})$, and LVI (LVI(-); $2.03 \pm 0.68, \mathrm{LVI}(+) ; 3.34 \pm 1.36, \mathrm{P}<$ 0.0001 , Figure. $4 \mathrm{E})$.

We investigated the correlation between Rac1 activity and Pak1 expression in tumor tissues. When Rac1 was used as an independent variable and Pak1 as a dependent variable, a positive correlation between them was observed $\left(\mathrm{r}^{2}=0.288, P<0.0001\right.$, Figure. $\left.5 \mathrm{~A}\right)$. However, no such correlation was observed in specimens of metastatic lymph nodes $\left(\mathrm{r}^{2}=0.307, P=0.2602\right.$, Figure. $\left.5 \mathrm{~B}\right)$.

\section{Prognostic influence of Rac1 activity and Pak1 expression}

The mean level of Rac1 activity was 2.72 ( \pm 1.72$)$. Patients were divided into two groups at this value, i.e., a high activity group (46 patients) and a low activity group (62 


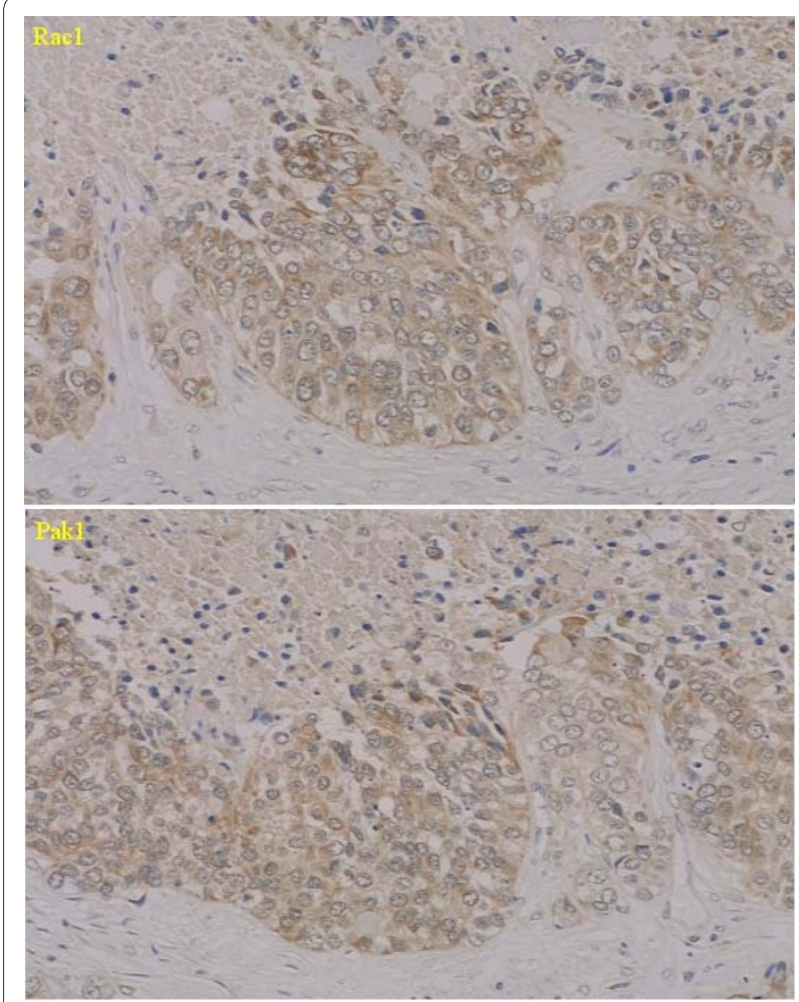

Figure $\mathbf{2}$ Immunohistochemistry for Rac1 (upper panel) and Pak1 (lower panel) proteins in Grade 3 carcinoma. Cytosolic compartments shows intensely brown staining in most of the cancer cells, displaying high Rac1 and Pak1 protein levels, but the nuclei of the cancer cells shows very weak staining.

patients), according to the method described previously [21-23]. Similarly, the mean level of Pak1 protein expression in the tumor tissues was 2.68 ( \pm 1.26$)$, so a high expression group (49 patients) and a low expression group (59 patients) were separated by using this as the cut-off value.

Kaplan-Meier plots of survival for patients with low versus high levels of Rac1 activity showed that increased Rac1 activity was associated with a shorter overall survival time $(P<0.0001$, Figure. 6A). High expression of Pak1 protein was also correlated with shorter overall survival $(P<0.0001$, Figure. $6 \mathrm{~B})$. Univariate analysis of overall survival with the Cox proportional hazards model revealed that tumor grade, $\mathrm{pT}$ stage, lymph node metastasis, LVI, active Rac1, and Pak1 were all significant variables (Table 1). However, multivariate analysis revealed that only LVI and Pak1 had an independent influence on overall survival.

With regard to disease-free survival of the patients who were NO MO at the time of nephroureterectomy (92 patients), the mean level of Rac1 activity and Pak1 expression in tumor tissue was $2.55 \pm 1.62$ and $2.43 \pm$ 1.09 , respectively. Comparison of Kaplan-Meier plots for the N0 M0 patients with low Rac1 activity (59 patients) versus high Rac1 activity (33 patients) showed that high Rac1 activity was associated with a shorter disease-free survival time $(P=0.001$, Figure. $6 C)$. Similarly, higher expression of Pak1 was a significant unfavorable factor for disease-free survival $(P<0.0001$, Figure. 6D). Although tumor grade, pT stage, LVI, active Rac1, and Pak1 were all significant factors by Cox univariate analysis, only LVI and Pak1 were independent variables according to multivariate analysis (Table 1).

With regard to the site of first postoperative recurrence among the 92 N0 M0 patients, the Rac1 activity and Pak1 protein levels in the primary tumor tissues of patients with retroperitoneal lymph node metastasis (PRLN) ( $\mathrm{n}=$ $14 ; 3.99 \pm 1.82,3.61 \pm 0.94$, respectively) and other organ metastases ( 3 of lung, one of liver, and one of bone; $6.35 \pm$ 2.35 and $4.49 \pm 1.90$, respectively) were significantly higher than the levels in patients with bladder recurrence (n = 18; $1.95 \pm 0.89, P<0.0001,2.32 \pm 1.02, P<0.01$, respectively, Figures. 3F, 4F). Furthermore, we divided this group into two subgroups to study the relationship between LVI and Rac1 activity or Pak1 expression (Figure. 7). When the PRLN and distant organs were the first site of recurrence, the primary tumors had LVI, while primary tumors without LVI showed no PRLN recurrence or distant metastasis. Among the LVI(+) group, both Rac1 activity and Pak1 expression in the primary tumors were higher in patients with RPLN and distant metastases than in those with bladder recurrence. The patients with bladder recurrence had higher tumor levels of Pak1 expression than those with no evidence of disease (NED), but Rac1 activity did not vary. On the other hand, in the LVI() group, both Rac1 activity and Pak1 expression were not different between the primary tumors of patients with bladder recurrence and those with NED. Interestingly, although Rac1 activity showed no difference between LVI(+) and LVI(-) tumors in patients with bladder recurrence, Pak1 expression was higher in the former tumors.

M-VAC therapy was performed for 16 patients with lymph node metastasis at the time of nephroureterectomy, 14 patients with postoperative lymph node involvement, and 5 patients with postoperative metastasis to other organs. All of these 35 patients eventually died of progressive cancer. Their primary tumors were all $\mathrm{LVI}(+)$ on pathological examination, as well as showing increased Rac1 activity and high Pak1 expression (data not shown).

\section{Discussion}

Rac1 and Pak1 have recently been shown to be key regulators of cancer cell signaling networks, and there are several lines of evidence linking Rac1 and Pak1 to the acquisition of migratory, invasive, and metastatic phenotypes $[7,9]$. In order to take into account possible inter- 


\section{A. Tumor and Lymph nodes in Rac 1}

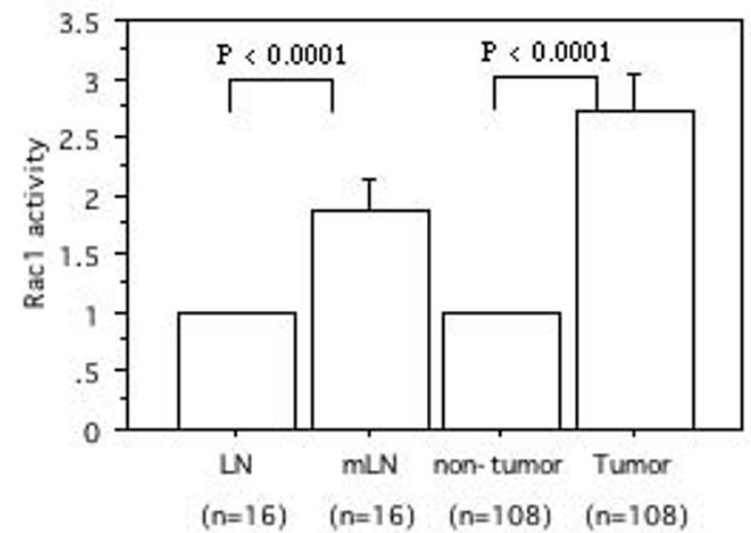

C. pT stage in Rac 1

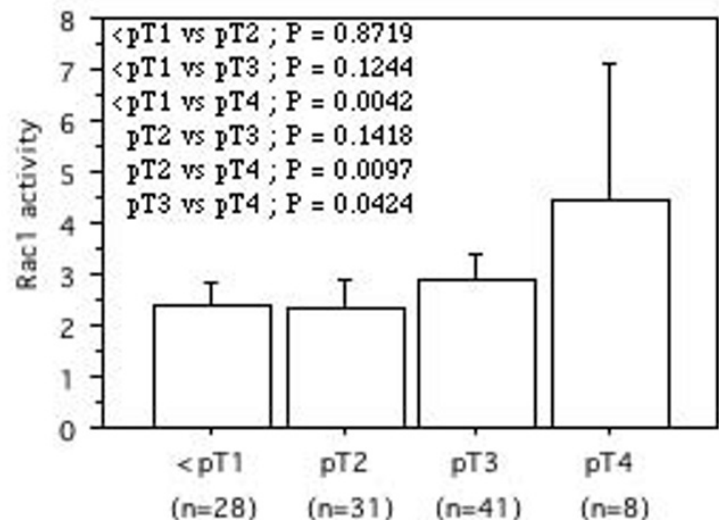

E. Lymphovascular invasion in Rac 1

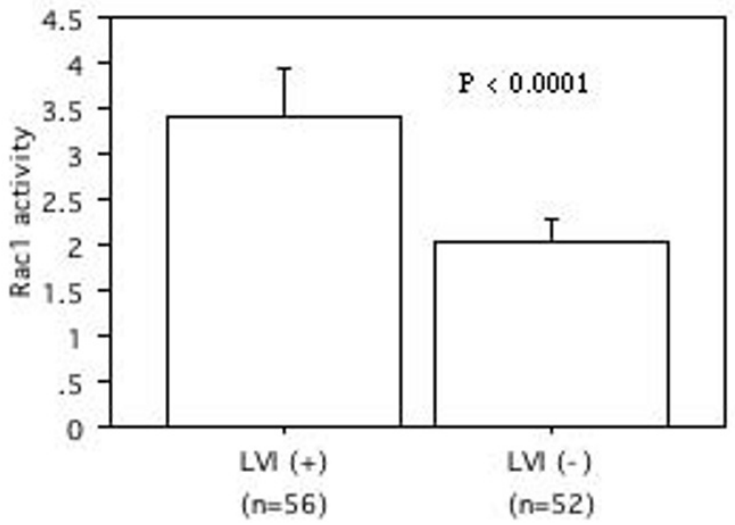

\section{B. Grade in Rac 1}

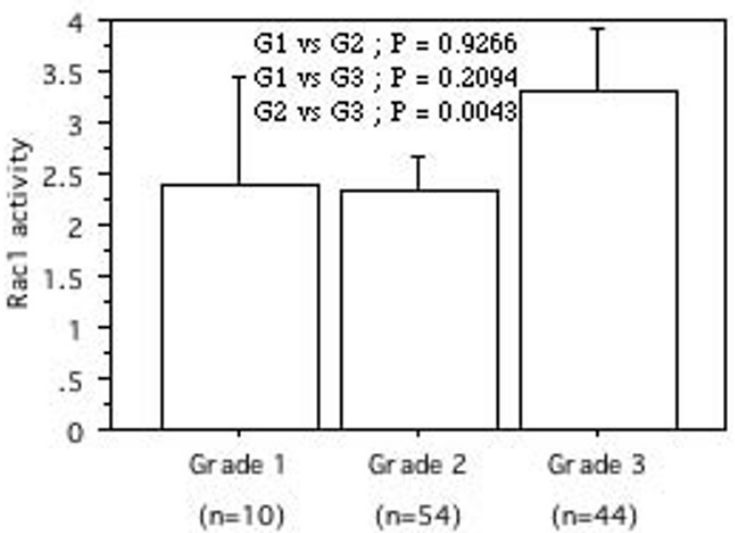

D. $\mathrm{pN}$ in Rac 1

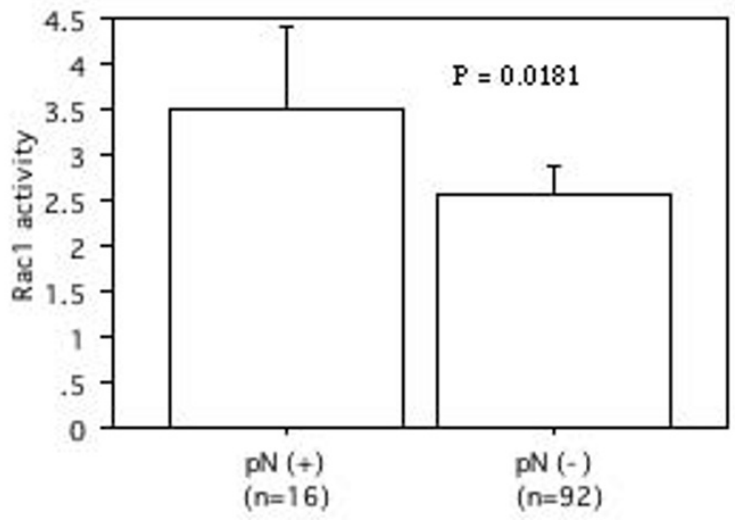

\section{F. Recurrence in NOMO cases in Rac 1}

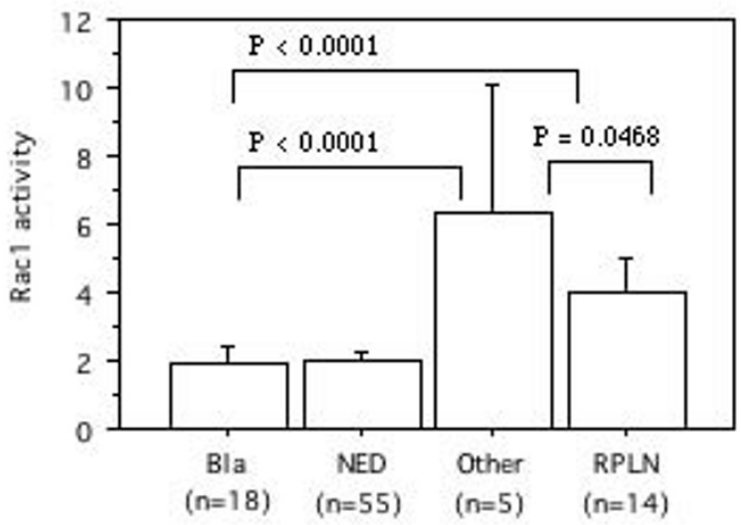

Figure 3 The relative expression levels of GTP-binding active Rac1 protein in tumor to those in corresponding non-tumor portion, which was set to 1.0. A; Expression in tumor, non-tumor, and lymph node tissues with metastasis (mLNs) and without (LN). B; Expression in Grade. C; Expression in $\mathrm{pT}$ stage. D; Expression in lymph node metastasis. $\mathrm{pN}(-)$ is $\mathrm{pNO}$. $\mathrm{pN}(+)$ is $\mathrm{pN1} 1-3$. E; Expression in lymphovascular invasion (LVI). F; Expression in first recurrence site. There were no difference between bladder and none of recurrence. The data show the $95 \%$ confidential interval.

individual variations of Rac1 activity and Pak1 protein expression in UC-UUT, we performed a comparison among paired samples of tumor tissue, metastatic lymph node tissue, and non-tumor tissue from the same patient. The present study showed that Rac1 activity and Pak1 expression were significantly increased in primary 
A. Tumor and Lymph nodes in Pak1

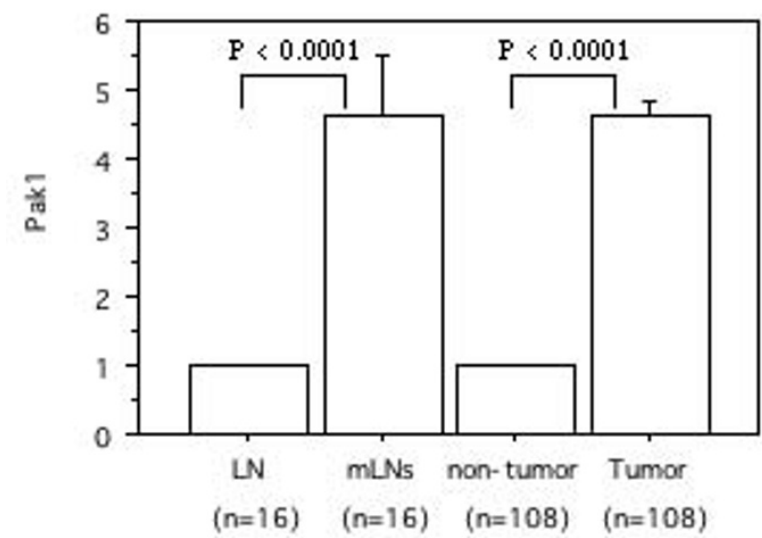

C. pT stage in Pak1

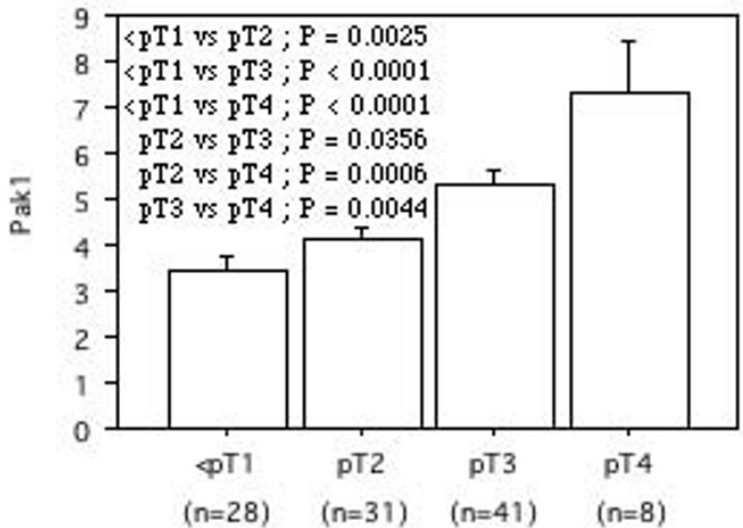

E. Lymphovascular invasion in Pak1

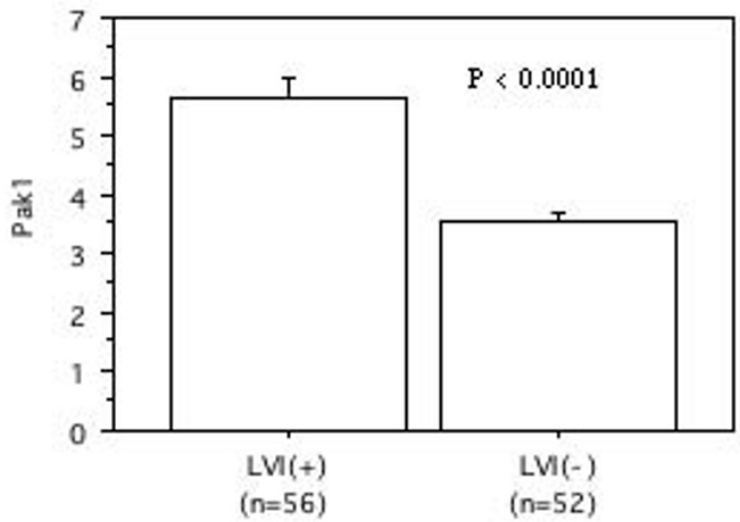

B. Grade in Pak1

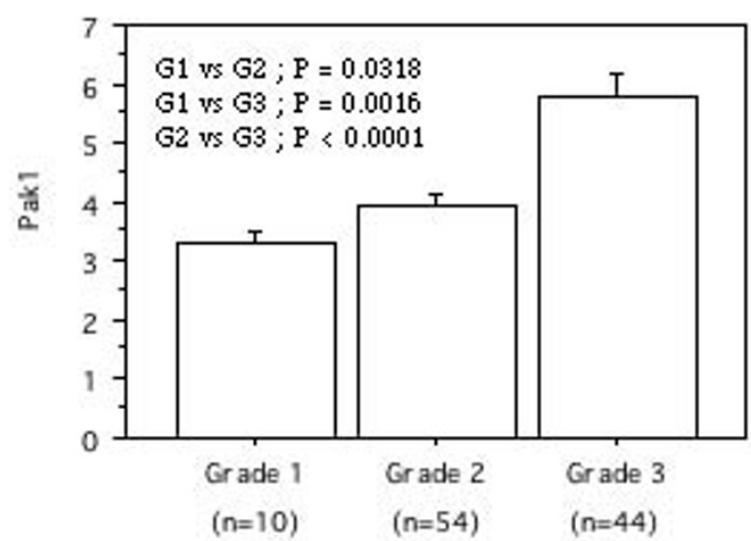

D. $\mathrm{pN}$ in Pak1

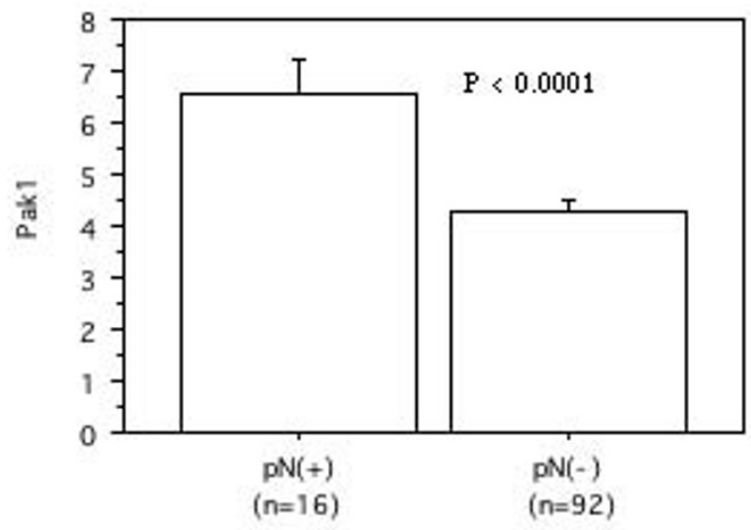

\section{F. Recurrence in NOMO cases in Pak1}

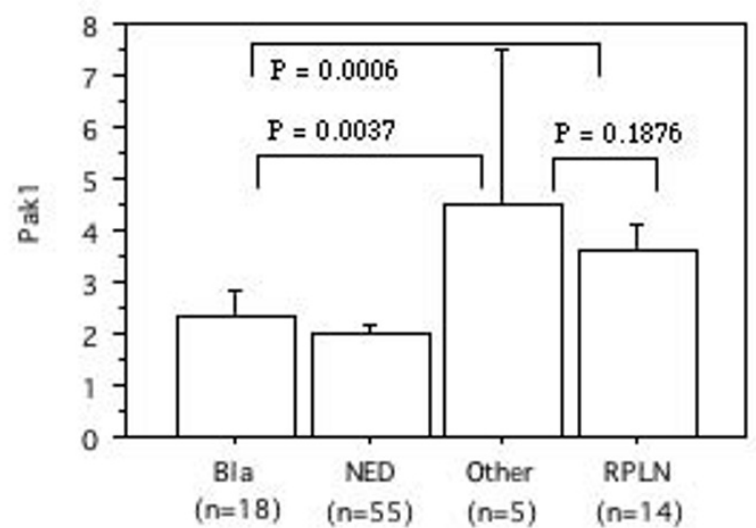

Figure 4 The relative expression levels of Pak1 protein in tumor to those in corresponding non-tumor portion, which was set to 1.0. $A$; Expression in tumor, non-tumor, and lymph node tissues with metastasis (mLNs) and without (LN). B; Expression in Grade. C; Expression in pT stage. D; Expression in lymph node metastasis. $\mathrm{pN}(-)$ is pN0. pN(+) is pN1-3. E; Expression in lymphovascular invasion (LVI). F; Expression in first recurrence site. There were no difference between bladder and none of recurrence. The data show the 95\% confidential interval. 


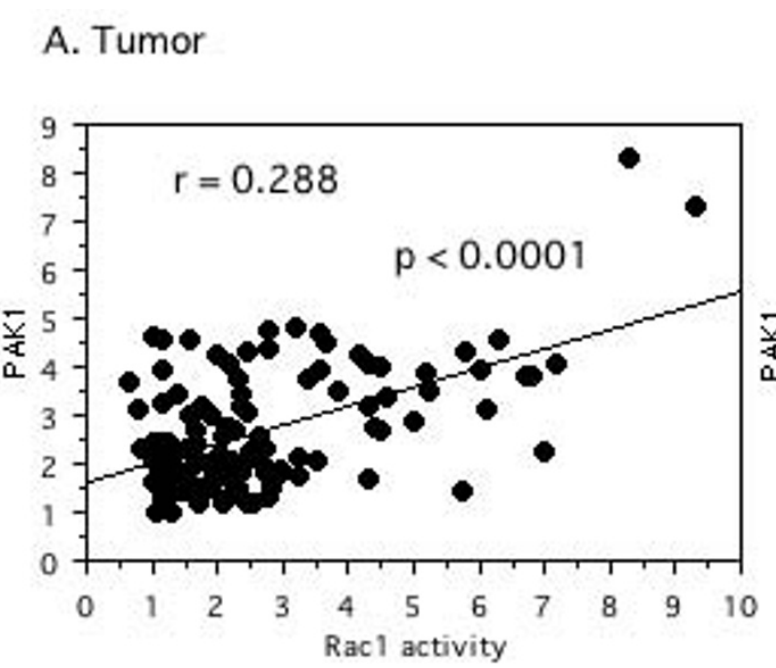

Figure 5 Spearman rank correlation coefficient relationship between expression levels of Rac1 activity and Pak1. $X$ axis is an independent variable. $Y$ axis is a dependent variable. A; Tumor tissues. B; Metastatic lymph node tissues.

tumors and metastatic lymph nodes compared with nontumor tissues. Also, an increase of Rac1 activity and Pak1 expression in the primary tumor was correlated with poorly differentiated cancer, local invasion, lymph node metastasis, LVI, and an unfavorable prognosis. To our knowledge, this is the first report about Rac1 and Pak1 in UC-UUT. Our data suggested that Rac1 and its downstream effector Pak1 may be involved in the progression of this cancer.

As well as our findings in patients with UC-UUT, overexpression of Rac1 and Pak1 has been reported in several other human cancers [7,9]. Moreover, an increase of Rac1 and Pak1 activity or overexpression have been observed in breast cancer tissues and metastatic lymph nodes $[24,25]$. In the present study, increased Rac1 activity and higher Pak1 expression in the primary tumors was related to muscle invasion and lymph node metastasis. Therefore, it is likely that Rac1 and Pak1 have a role in determining the local invasive and metastatic potential of various human cancers.

Regarding the site of initial postoperative recurrence in patients who were $\mathrm{pT}_{\text {any }} \mathrm{pNO} \mathrm{MO}$ at the time of nephroureterectomy, patients with postoperative lymph node recurrence had a worse prognosis than those with bladder recurrence, probably because many bladder cancers were detected at the superficial stage by active surveillance. In the present study, LVI, Pak1 activity, Rac1, pT stage, and tumor grade were related to postoperative recurrence according to univariate analysis, with both LVI and Pak1 still being significant determinants according to multivariate analysis. As shown in Figure 7, Rac1 activity and Pak1 expression were higher in the primary
B. metastatic lymph node

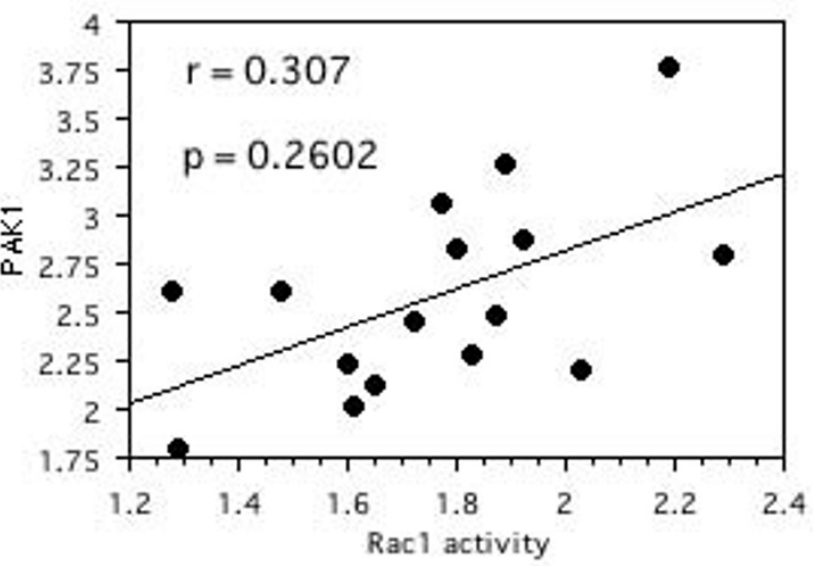

tumors of patients with postoperative lymph node metastasis than in those of patients with bladder recurrence from the LVI(+) group, but not the LVI(-) group. Pak1 expression was higher in the tumors of patients with bladder recurrence than in recurrence-free patients from the LVI(+) group, but Rac1 did not differ between them. Moreover, all patients with lymph node involvement at nephroureterectomy had LVI(+) tumors on pathological examination. Therefore, LVI might be an important step along the road to lymph node metastasis. Primary tumors with LVI(+) and lymph node metastasis showed an increase of Rac 1 activity and Pak 1 expression, while metastatic lymph node tissues showed higher Rac1 activity and Pak1 expression than normal lymph nodes, indicating that Rac1 and Pak1 are involved in tumor metastasis. However, it is unclear whether Rac1 and Pak1 play a similar role in lymph node metastasis and bladder recurrence. It is well known that urothelial cancer often behaves like a field change disease, with multiple occurrences and recurrences due to implantation and migration, making it difficult to determine whether a recurrent tumor has been caused by tumor cell implantation, migration, or multifocal carcinogenesis [26]. A recent molecular study revealed that UC-UUT might be less genetically stable than bladder tumors [27]. Therefore, an increase of Rac1 activity and upregulation of Pak1 expression might play a role in lymph node metastasis of UC-UTT after nephroureterectomy, rather than contributing to bladder recurrence. The differences of molecular mechanisms between LVI and lymph node metastasis or bladder recurrence need to be investigated further. On the other hand, a previous study of bladder cancer showed that 


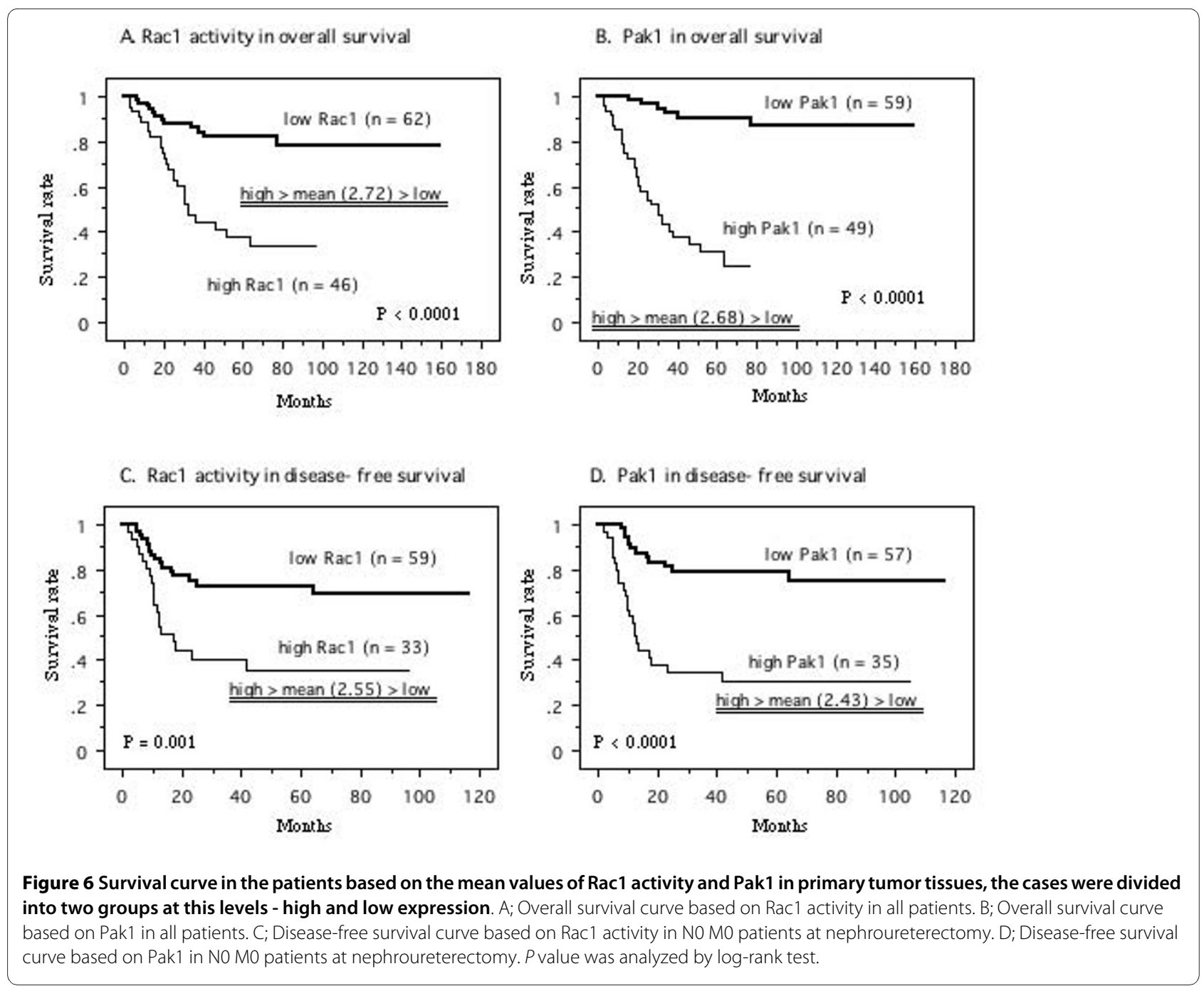

high Pak1 expression was associated with a higher risk of recurrence, even in patients with low grade/stage tumors [28]. Taken together, therefore, it is likely that Pak1 and Rac1 both play an important role in the invasion, metastasis, and recurrence of urothelial cancer.

Although Pak1 is well known as a downstream effector of Rac1, there have been few simultaneous analyses of Rac1 and Pak1 expression in human tumor tissues. Our study showed a positive correlation between Rac1 activity and Pak1 expression in tumor tissue, while no such relation was observed in metastatic lymph node tissue. In contrast to investigation of Rac1 activity, we only measured Pak1 protein expression, but we could still determine the approximate relation between the two molecules. As shown in Figure 5, there was a positive correlation in tumor tissues, but the absolute correlation was fairly weak and no correlation was found in metastatic lymph nodes. Pak1 is the best-characterized downstream effector of Rac1, but it is also an important convergence point for many signaling pathways (including small
GTPases and several tyrosine kinases) that are often activated in cancer cells [9-11]. Therefore, several oncogenic pathways may act through Pak1 to promote cancer progression, so that Pak1 protein expression had a greater impact on overall and recurrence-free survival than Rac1 activity in the present study. We did not assess Pak1 activity in this study, so its activity in tumor tissues needs to be elucidated in the future.

Cell migration is central to metastasis by malignant tumors [3]. Members of the Rho small GTPases family regulate formation of stress fibers, focal adhesions, and cell migration through reorganization of the actin cytoskeleton [6]. Several lines of evidence have directly linked Rac1 and Pak1 to acquisition of a migratory, invasive, and metastatic phenotype and to a variety of processes that occur in tumors, including cell transformation, survival, invasion, metastasis, and angiogenesis [7,9]. Our findings suggested that Rac1 and Pak1 were associated with LVI and RPLN, as well as distant metastasis of UC-UUT. 
Table 1: Cox regression analysis for various potential prognostic factors in survival

\begin{tabular}{|c|c|c|c|c|c|c|c|c|c|c|c|}
\hline \multirow[b]{2}{*}{ Variable } & \multirow[b]{2}{*}{$\begin{array}{c}\text { Unfavorable/ } \\
\text { favorable } \\
\text { characteristics }\end{array}$} & \multicolumn{4}{|c|}{ Overall survival in all patients } & \multirow[b]{2}{*}{$P$ value } & \multirow[b]{2}{*}{$\begin{array}{l}\text { No. of } \\
\text { Patients }\end{array}$} & \multicolumn{3}{|c|}{$\begin{array}{l}\text { Disease-free survival in NO MO patients at } \\
\text { nephroureterectomy }\end{array}$} & \multirow[b]{2}{*}{$P$ value } \\
\hline & & $\begin{array}{l}\text { No. of } \\
\text { Patients }\end{array}$ & Analysis & $\begin{array}{l}\text { Relative } \\
\text { risk }\end{array}$ & $\begin{array}{c}95 \% \\
\text { confidential } \\
\text { interval }\end{array}$ & & & Analysis & $\begin{array}{l}\text { Relative } \\
\text { risk }\end{array}$ & $\begin{array}{c}95 \% \\
\text { confidential } \\
\text { interval }\end{array}$ & \\
\hline & & & Univariate $(U)$ & 2.626 & $1.486-4.641$ & 0.0009 & & Univariate $(U)$ & 1.947 & $1.117-3.392$ & 0.0187 \\
\hline \multirow[t]{3}{*}{ Grade } & $3 / 2 / 1$ & $44 / 54 / 10$ & & & & & $32 / 51 / 9$ & & & & \\
\hline & & & Multivariate (M) & 1.008 & $0.488-2.083$ & 0.6126 & & $\begin{array}{l}\text { Multivariate } \\
\text { (M) }\end{array}$ & 1.047 & $0.427-1.678$ & 0.6332 \\
\hline & & & $\mathrm{U}$ & 3.138 & $2.065-4.768$ & $<0.0001$ & & U & 1.905 & $1.308-2.774$ & 0.0008 \\
\hline \multirow[t]{3}{*}{ рт } & $4 / 3 / 2 / 1>$ & $8 / 41 / 31 / 28$ & & & & & $3 / 37 / 26 / 26$ & & & & \\
\hline & & & M & 1.499 & $0.837-2.526$ & 0.1454 & & M & 1.291 & $0.479-1 / 302$ & 0.3551 \\
\hline & & & U & 3.049 & $2.005-4.636$ & $<0.0001$ & & & & & \\
\hline \multirow[t]{3}{*}{$\mathbf{p N}$} & $(+) /(-)$ & $16 / 92$ & & & & & & & & & \\
\hline & & & M & 1.48 & $0.894-2.448$ & 0.1271 & & & & & \\
\hline & & & U & 11.024 & $5.550-21.896$ & $<0.0001$ & & U & 10.298 & $4.737-22.388$ & $<0.0001$ \\
\hline \multirow[t]{3}{*}{ LVI } & $(+) /(-)$ & $56 / 52$ & & & & & $40 / 52$ & & & & \\
\hline & & & M & 6.923 & $2432-19.707$ & $<0.0001$ & & M & 9.954 & $4.093-24.204$ & $<0.0001$ \\
\hline & & & U & 4.363 & $2.188-8.702$ & $<0.0001$ & & U & 2.917 & $1.494-5.694$ & 0.0017 \\
\hline \multirow[t]{3}{*}{ Rac1 } & high/low & $46 / 62$ & & & & & $33 / 59$ & & & & \\
\hline & & & M & 1.87 & $0.863-4.094$ & 0.1223 & & M & 1.323 & $0.627-2.791$ & 0.4621 \\
\hline & & & U & 12.633 & $4.869-32.776$ & $<0.0001$ & & U & 4.814 & $2.384-9.721$ & $<0.0001$ \\
\hline \multirow[t]{2}{*}{ Pak1 } & high/low & $49 / 59$ & & & & & $35 / 57$ & & & & \\
\hline & & & $M$ & 3.635 & $1.230-10.741$ & 0.0196 & & $M$ & 3.526 & $1.564-7.946$ & 0.0024 \\
\hline
\end{tabular}


A. Rac1 activity in primary tumors regarding with recurrence site in NOMO cases

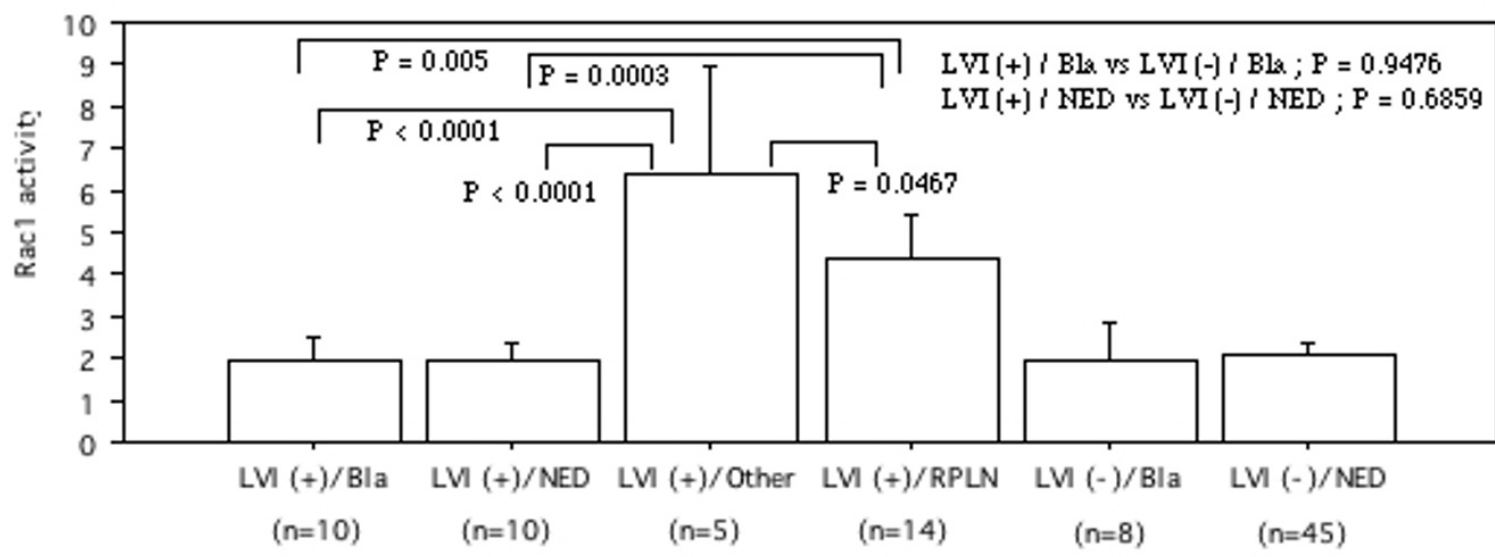

B. Pak 1 expression in primary tumors regarding with recurrence site in NOMO cases

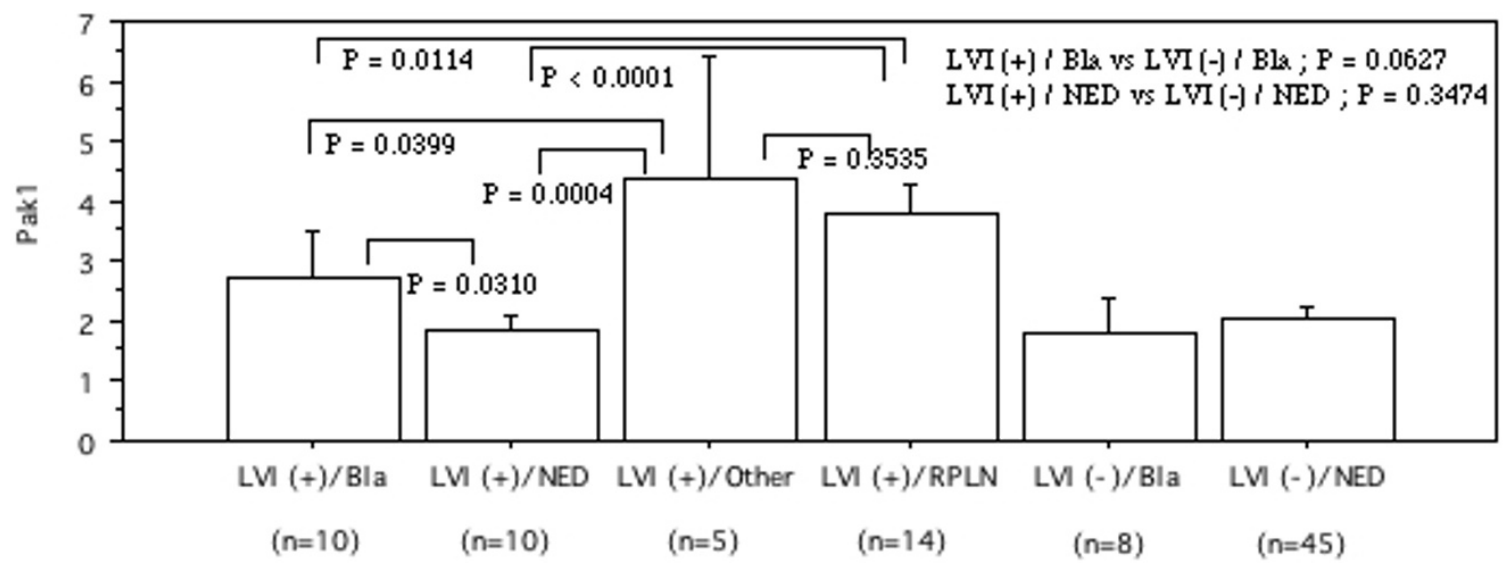

Figure 7 Rac1 activity (A) and protein levels of Pak1 (B) regarding with postoperative first recurrence site among N0 M0 cases (92 patients). The data show the 95\% confidential interval.

The renal pelvis and ureter have thin walls and a rich lymphatic drainage [1], so many patients present with local invasion and/or lymph node metastasis, while delay in making the initial diagnosis is correlated with a higher stage at presentation. Preoperative staging by CT/MRI was very useful for detecting patients with local invasion and/or lymph node metastasis in the present study, but the prognosis of such patients was poor (Table 1). Although systemic M-VAC therapy reduced the tumor burden of our patients with urothelial cancer, the prognosis was worse than we expected [17]. In the present study, M-VAC therapy was performed as adjuvant chemotherapy for the 35 patients who showed lymph node or distant metastasis at surgery or during postoperative surveillance, but all of these patients died of their cancer.
These 35 tumors were all $\mathrm{LVI}(+)$ and showed increased Rac1 activity and high Pak1 expression. Furthermore, an increase of Rac1 activity and a higher Pak1 expression were associated with a shorter overall survival time in all patients and shorter postoperative disease-free survival in $\mathrm{pT}_{\text {any }} \mathrm{pNO} \mathrm{M} 0$ patients, indicating that Rac1 activity and Pak1 expression may be useful prognostic indicators for UC-UTT. These findings suggests that patients with $\mathrm{LVI}(+)$ tumors that have higher Rac1 activity and Pak1 expression are at more risk of developing postoperative RPLN or distant metastases in comparison to patients without these markers. Therefore, we need to assess the potential of chemotherapy for patients who have $\mathrm{pT}_{\text {any }} \mathrm{pNO} \mathrm{M} 0$ tumors that are $\mathrm{LVI}(+)$ with increased Rac1 activity and Pak1 expression to prevent RPLN recurrence 
or distant metastasis by performing a randomized study in the future. In premenopausal breast cancer patients, Pak1 overexpression has been closely linked with tamoxifen resistance of tumors [29]. On the other hand, several low molecular weight inhibitors have been shown to interfere with Pak1 kinase activity or function [9]. Therefore, the Rac1-Pak1 pathway might be a potential therapeutic target for the prevention of tumor invasion and metastasis by inhibition of this signaling pathway. Accordingly, we should examine the effects of various inhibitors using cell lines or tumor tissue samples. Furthermore, as the Rac family has three isoforms and the Pak family has six isoforms, each isoform may play a different role. Therefore, it is necessary to investigate each of these isoforms in human cancers, and the information thus obtained may shed new light on treatment strategies for UC-UTT and other tumors.

\section{Conclusion}

In the present study, increased Rac1 activity and increased expression of Pak1 (a major downstream effector) were associated with poorly differentiated tumors, local invasion, LVI, lymph node metastasis, and an unfavorable prognosis. Our findings suggest that the Rac1Pak1 pathway may be related to the progression of UCUUT and that these molecules may be indicators for this disease.

\section{Competing interests}

The authors declare that they have no competing interests.

\section{Authors' contributions}

T.K.* and H.S. initiated the study, participated in its design and coordination, carried out the study, performed the statistical analysis, and drafted the manuscript. K.N., N.F., T.K., H.A. and T.O. carried out the study. K-I.Y. participated in the design of the study and helped to draft the manuscript. All authors read and approved the final manuscript.

\section{Acknowledgements}

The authors are special grateful to Hitomi Yamazaki for her excellent technique in this study.

\section{Author Details}

'Department of Urology, Dokkyo Medical University, Mibu, Tochigi, Japan, 2Department of Molecular and Cell Biology, Dokkyo Medical University, Mibu, Tochigi, Japan and ${ }^{3}$ Department of Anatomic and Diagnostic Pathology, Dokkyo Medical University, Mibu, Tochigi, Japan

Received: 25 September 2009 Accepted: 28 April 2010 Published: 28 April 2010

\section{References}

1. Munoz JJ, Ellison LM: Upper tract urothelial neoplasms: incidence and survival during the last 2 dacades. J Urol 2000, 164:1523-1525.

2. Hall MC, Womack S, Sagalowsky AL, Carmody T, Erickstad MD, Roehrborn CG: Prognostic factors, recurrence, and survival in transitional cell carcinoma of the upper urinary tract: a 30-year experience in 252 patients. Urology 1998, 52:594-601.

3. Quigley JP, Armstrong PB: Tumor cell intravasation elu-cidated; The chick embryo opens the window. Cell 1998, 94:281-284.

4. Kikuchi E, Horiguchi Y, Nakashima J, Hatakeyama N, Matsumoto M, Nishiyama T, Murai M: Lymphovascular invasion independently predicts increased disease specific survival in patients with transitional cell carcinoma of the upper urinary tract. J Urol 2005, 174:2120-2124.

5. Ozsahin M, Zouhair A, Villa S, Storme G, Chauvet B, Taussky D, Gouders D, Ries G, Bontemps P, Coucke PA, Mirimanoff RO: Prognostic factors in urothelial renal pelvis and ureter tumors: a multicentre Rare Cancer Network study. Eur J Cancer 1999, 35:738-743.

6. Etienne-Manneville S, Hall A: Rho GTPases in cell biology. Nature 2002, 420:629-635.

7. Sahai E, Marshall CJ: Rho-GTPases and cancer. Nature Rev Cancer 2002, 2:133-142.

8. Kamai T, Kawakami S, Koga F, Arai G, Takagi K, Arai K, Tsujii T, Yoshida K-I: RhoA is associated with invasion and lymph node metastasis in upper urinary tract cancer. BJU Int 2003, 91:234-238.

9. Kumar R, Gururaj AE, Barnes CJ: P21-activated kinases in cancer. Nature Rev Cancer 2006, 6:459-471.

10. Mira JP, Berard V, Groffen J, Sanders LC, Knaus UG: Endogeneos, hyperactive Rac3 controls proliferation of breast cancer cells by a p21activated kinase-dependent pathway. Proc Natl Acad Sci USA 2000, 97:185-189

11. Balasenthil S, Sahin AA, Barnes CJ, Wang RA, Pestell RG, Vadlamudi RK: p21-activated kinase-1 signalingmediates cyclin D1 expression in mammary epithelial and cancer cells. J Biol Chem 2004, 279:1422-1428.

12. Zohn IM, Campbell SL, Khosravi-Far R, Rossman KL, Der CJ: Rho family proteins and Ras transformation: the RHOad less traveled gets congested. Oncogene 1998, 17:1415-1438.

13. Nobes CD, Hall A: Rho, Rac, and Cdc42 GTPases regulate the assembly of multimolecular focal complexes associated with actin stress fibers, lamellipodia, and filopodia. Cell 1995, 81:53-62.

14. Gao Y, Dickerson JB, Guo F, Zheng J, Zheng Y: Rational design and charaterization of a Rac GTPase-specific small molecular inhibitor. Proc Natl Acad SciUSA 2004, 101:7618-7623.

15. Suwa H, Ohshio G, Imamura T, Watanabe G, Arii S, Imamura M, Narumiya $\mathrm{S}$, Hiai H, Fukumoto M: Overexpression of the rhoC gene correlates with progression of ductal adenocarcinoma of the pancreas. Br J Cancer 1998, 77:147-152.

16. Sobin $\mathrm{H}$, Wittekind $\mathrm{CH}$, editors: International union against cancer. UICC. In TNM classification of malignant tumors 6th edition. New York, Wiley-Liss; 2002

17. Conner JP, Rpoportm F, Olsson CA, Sawczuk IS, Benson MC: Long-term follow-up patients treated with methotrexate, vinblastine, doxorubicin and cisplatin (M-VAC) for transitional cell carcinoma of urinary bladder: Cause for concern. Urology 1990, 34:353-356.

18. Nakanishi K, Kamai T, Mizuno T, Arai K, Yamanishi T: Expression of RhoA mRNA and activated RhoA in urothelium and smooth muscle, and effects of a Rho-kinase inhibitor on contraction of the porcine urinary bladder. Neurourol Urodynam 2009, 28:521-528.

19. Habas R, Dawid IB, He X: Coactivation of Rac and Rho by Wnt/Frizzled signaling is required for vertebrate gastrulation. Genes Dev 2003, 17:295-307.

20. Alstergren R, Zhu B, Glougauer M, Mak TW, Ellen RP, Sodek J: Polarization and directed migration of murine meutrophils is dependent on cell surface expression of CD44. Cell Immunol 2004, 231:146-157.

21. Abe H, Kamai T, Shirataki H, Oyama T, Arai K, Yoshida K-l: High expression of Ran GTPase is associated with local invasion and metastasis of human clear cell renal cell carcinoma. Int J Cancer 2008, 122:2391-2397.

22. Kamai T, Tsujii T, Arai K, Takagi K, Asami H, Ito Y, Oshima H, Yoshida K-I: Significant association of Rho/ROCK pathway with invasion and metastasis of bladder cancer. Clin Cancer Res 2003, 9:2632-2641.

23. Fritz G, Just I, Kaina B: Rho GTPase over-expressed in human tumors. Int J Cancer 1999, 81:682-687.

24. Fiegen D, Haeusler LC, Blumenstein L, Herbrand U, Dvorsky R, Vetter IR, Ahmadian MR: Alternative splicing of Rac1 generates Rac1b, a selfactivating GTPase. J Biol Chem 2004, 279:4743-4749.

25. Schnelzer A, Prechtel D, Knaus U, Dehne K, Gerhard M, Graeff H, Harbeck $\mathrm{N}$, Schmitt M, Lengyel E: Rac1 in human breast cancer: overexpression, mutation analysis, and characterization of a new isoform, Rac1b. Oncogene 2000, 19:3013-3020

26. Messing ED: Urothelial tumors of the bladder. In Campbell-Walsh Urology 9th edition. Edited by: Wein AJ, Kavoussi LR, Novick AC, Partin AW, Peters CA. Philadelphia: Saunders Elsevier; 2007:2407-2446. 
27. Takahashi T, Kakehi Y, Mitsumori K, Akao T, Terachi T, Ogawa O, Habuchi T: Distinct microsatellite alterations in upper urinary tract tumors and subsequent bladder tumors. J Urol 2001, 165:672-677.

28. Ito M, Nishiyama H, Kawanishi H, Matsui S, Guilford P, Reeve A, Ogawa O: P21-activated kinase 1: a new molecular marker for intravesical recurrence after transurethral resection of bladder cancer. Clin Cancer Res 2007, 178:1073-1079.

29. Holm C, Rayala S, Jirstrom K, Stal O, Kumar R, Landberg G: Association between Pak1 expression and subcellular localization and tamoxifen resistance in breast cancer patients. J Natl Cancer Inst 2006, 98:671-680.

\section{Pre-publication history}

The pre-publication history for this paper can be accessed here: http://www.biomedcentral.com/1471-2407/10/164/prepub

\section{doi: 10.1186/1471-2407-10-164}

Cite this article as: Kamai et al., Increased Rac1 activity and Pak1 overexpression are associated with lymphovascular invasion and lymph node metastasis of upper urinary tract cancer BMC Cancer 2010, 10:164

Submit your next manuscript to BioMed Central and take full advantage of:

- Convenient online submission

- Thorough peer review

- No space constraints or color figure charges

- Immediate publication on acceptance

- Inclusion in PubMed, CAS, Scopus and Google Scholar

- Research which is freely available for redistribution 